\title{
Égalité des chances en École d'Ingénieur : \\ Retour sur 11 années d'expérimentation ...
}

Agnès Volpi, Michel Robert, Marc-Hubert Depret,

Céline Bouby, Tarak Ben Zineb, Gérard Jeandel, Marc Galiana

prénom.nom@esstin.uhp-nancy.fr

Nancy Université, ESSTIN, 2 rue Jean Lamour, 54519 Vandœuvre Les Nancy Cedex

RÉSUMÉ : Cette communication est une synthèse des actions menées au sein d'une école d'ingénieur universitaire afin de promouvoir l'égalité des chances auprès des collégiens et lycéens appartenant à des "milieux défavorisés" d'une part, des jeunes femmes (qui sont notoirement sous-représentées en école d'ingénieur) d'autre part.

Mots clés : égalité des chances, ouverture sociale

\section{CONTEXTE}

L'ESSTIN (École Supérieure des Sciences et Technologies de l'Ingénieur de Nancy) est une composante de l'Université Henri Poincaré. C'est une école d'ingénieurs en 5 ans qui recrute la majorité de ses futurs diplômés à l'issue du baccalauréat. Avec un taux de boursiers supérieur à $35 \%$. l'ESSTIN a, depuis de nombreuses années, anticipé l'objectif fixé par les tutelles "d'accueillir [dans les grandes écoles] $30 \%$ de boursiers" et ce, sans instaurer de quotas ; l'école a ainsi apporté une contribution positive à la polémique stérile qui s'est développée courant janvier 2009 [1] [2]. En effet, s'il en était besoin, les tableau et graphique cidessous - extraits respectivement de [3] [4] justifieraient la nécessité de mener des actions visant à promouvoir l'égalité des chances.

\begin{tabular}{|c|c|c|c|c|c|c|c|}
\hline $\begin{array}{r}\text { Agricul } \\
\text { art } \\
\text { commerça } \\
\text { chefs d'entr }\end{array}$ & $\begin{array}{l}\text { Ilteurs, } \\
\text { tisans, } \\
\text { ants et } \\
\text { eprise }\end{array}$ & $\begin{array}{r}\text { Cadres et } \\
\text { professions } \\
\text { intellectuelles } \\
\text { supérieures }\end{array}$ & $\begin{array}{r}\text { Professions } \\
\text { intermédiaires }\end{array}$ & Employés & Ouvriers & $\begin{array}{c}\text { Retraités et } \\
\text { inactifs }\end{array}$ & $\begin{array}{l}\text { Non ren- } \\
\text { seignés }\end{array}$ \\
\hline Disciplines générales et de santé & 8,3 & 31,6 & 14,0 & 13,1 & 9,8 & 12,3 & 11,1 \\
\hline UT & 10,9 & 28,5 & 17,6 & 16,8 & 14,2 & 8,3 & 3,7 \\
\hline Grands établissements & 9,0 & 48,2 & 9,6 & 6,9 & 3,8 & 10,9 & 11,5 \\
\hline STS & 12,3 & 15,2 & 14,5 & 16,4 & 20,5 & 16,9 & 4,2 \\
\hline CPGE & 10,2 & 49,3 & 13,0 & 9,2 & 5,7 & 9,5 & 3,1 \\
\hline Formations comptables non universitaires & 11,0 & 15,7 & 11,6 & 14,1 & 14,7 & 13,6 & 19,3 \\
\hline Universités de technologie et INP & 9,4 & 48,3 & 15,5 & 8,5 & 6,6 & 8,4 & 3,3 \\
\hline Autres formations d'ingénieurs non universitaires (1 & 1) 11,6 & 45,3 & 12,7 & 6,9 & 4,3 & 5,4 & 13,7 \\
\hline Écoles de commerce, gestion, vente et comptabilité & 14,9 & 37,9 & 7,2 & 6,0 & 2,2 & 4,7 & 27,1 \\
\hline Écoles paramédicales et sociales & 11,9 & 18,9 & 12,1 & 19,0 & 17,6 & 2,1 & 18,4 \\
\hline Total & 9,7 & 30,0 & 13,5 & 13,0 & 10,7 & 10,9 & 12,2 \\
\hline Population française (3) & 8 & 11 & 16 & 11 & 25 & 29 & \\
\hline
\end{tabular}

(1) Y compris les formations d'ingénieurs en partenariat. (2)Y compris écoles supérieures d'architecture, de journalisme et de communication. (3) Source INSEE : recensement de la population 1999. Les données concernant les grands établissements, les Écoles supérieures artistiques et culturelles, les autres formations d'ingénieurs non universitaires, les formations comptables non universitaires, les écoles de commerce, gestion, vente et comptabilité sont celles de 2007. Les données concernant les écoles paramédicales et sociales sont celles de 2006.

fig. 1 : Répartition en 2008-2009 des étudiants français selon la Profession et Catégorie Sociale du chef de famille (\%) (France métropolitaine + DOM) d'après [3] 
La figure 1 met en évidence la surreprésentation de certaines catégories sociales dans l'enseignement supérieur par rapport au pourcentage qu'elles représentent dans la population française. Les "démonstrations" de Pierre Bourdieu et de JeanClaude Passeron [5] - qui, dès les années 1960, ont mis en évidence, qu'en fonction de leur origine sociale, les étudiants n'ont pas tous les mêmes facilités d'accès à la culture et donc pas les mêmes chances de réussite scolaire - sont plus que jamais d'actualité aujourd'hui. Cette analyse est réactualisée dans [6] ; elle prend ainsi en compte l'évolution du nombre d'étudiants - 851600 en 1970 contre 2231700 en 2008 - phénomène parfois qualifié de massification.

Le graphique présenté en figure 2 met en évidence les déséquilibres, en termes de disciplines et de filières, liés au genre. Les données présentées confirment que, sur une période de 15 années, il n'y a pas de "renversement" de tendance ; les jeunes femmes privilégient toujours pour leur choix de filières, dans l'enseignement supérieur, les formations qui préparent aux métiers de la santé ou de l'enseignement. Malgré la forte demande exprimée par les milieux professionnels, bien que les statistiques mettent en évidence un gain de quelques pourcents, elles sont encore minoritaires dans la plupart des écoles d'ingénieurs. Pour ce qui concerne l'ESSTIN, le taux de féminisation n'est que de $17 \%$ alors que ce taux est de $27 \%$ pour l'ensemble des écoles d'ingénieurs françaises [3] [15].

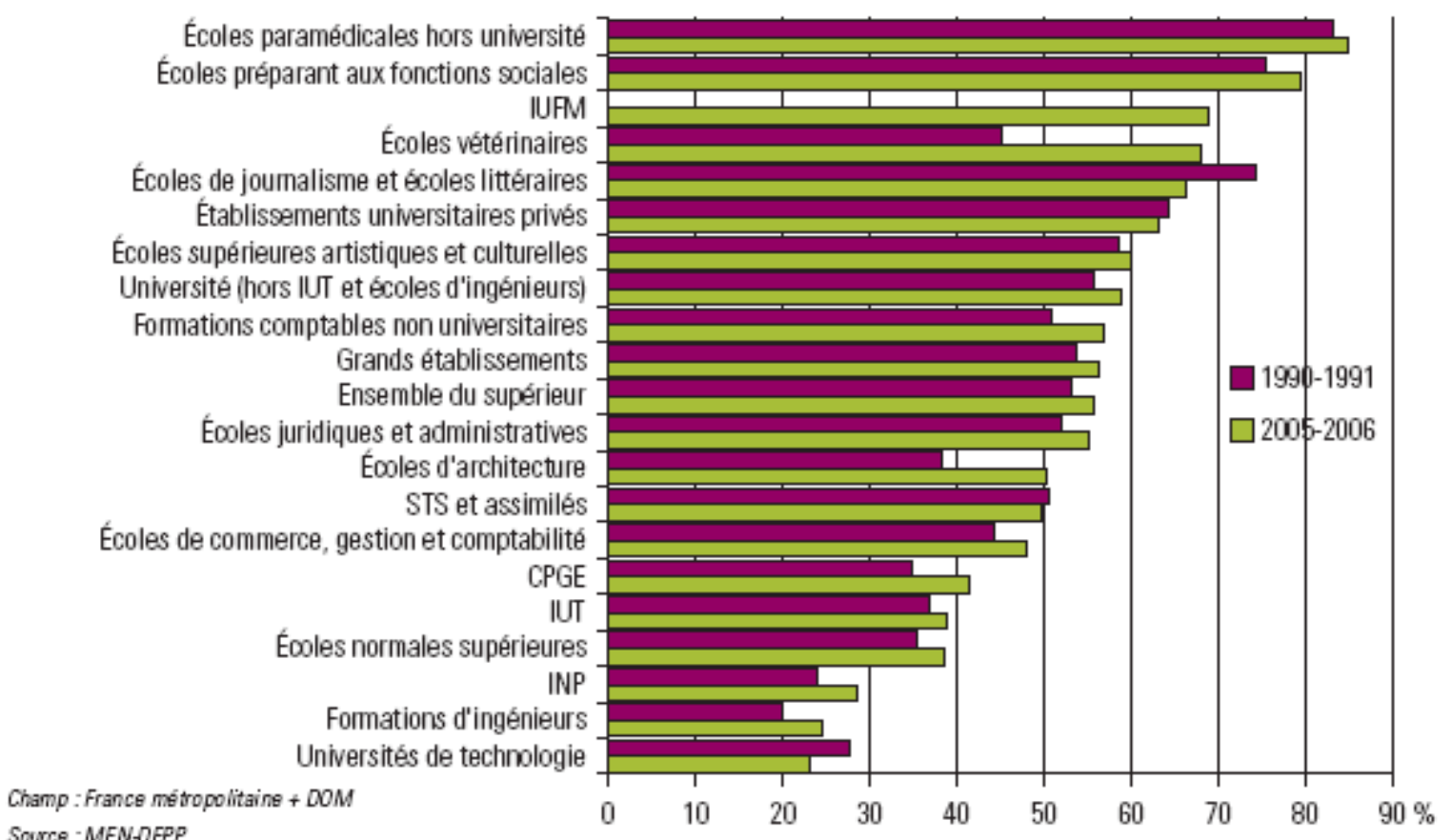

fig. 2 : Pourcentage de filles dans l'enseignement supérieur d'après [4]

L'ESSTIN a choisi de mettre en œuvre diverses actions citoyennes, avec un double objectif :

- apporter une contribution réelle aux actions visant à diversifier les origines des étudiants,

- développer, par une approche "sur le terrain", diverses compétences indispensables à un futur ingénieur (ouverture, travail en équipe, autonomie, créativité, transmission de savoir, ...)

Ces actions ont été rendues possibles grâce au soutien financier de l'État, des collectivités locales, de diverses associations et entreprises. Les forces vives impliquées sont principalement les élèves de
l'Ecole, accompagnés par des enseignants volontaires. Ces actions sont conduites au travers d'actes pédagogiques validés dans le cursus, les PIVE (Projet d'Implication dans la Vie de l'Ecole) et les projets de "responsabilité globale" (dit projet "citoyen").

Le règlement de scolarité de l'ESSTIN pour l'année 2010-2011 intègre une U.E. obligatoire "Responsabilité Globale", que les élèves doivent valider au moins une fois entre les semestres S3 et S8 ; U.E. qui permet de capitaliser entre 4 et potentiellement 14 crédits ECTS. Le règlement de scolarité précise également : 
- qu'en ce qui concerne les PIVE, un rapport écrit de 20 pages maximum, complété par une soutenance orale seront les éléments qui permettront de valider ou non l'U.E. associée et l'attribution d'ECTS,

- qu'en ce qui concerne l'activité Projet Citoyen, les élèves directement admis à l'ESSTIN en semestre S5, après l'obtention d'un BTS/DUT, doivent effectuer durant l'année de leur admission un "Projet Citoyen". Ce projet fait l'objet d'un rapport d'activité qui est soutenu oralement devant le jury ad hoc, évalué, noté et pris en considération dans la formation et autorisera l'attribution d'ECTS.

\section{PUBLICS CIBLES ET OBJECTIFS}

Les actions sont organisées autour de 2 thématiques :

- "Égalité des chances - Ouverture sociale", à destination des collégiens et lycéens appartenant à des "milieux défavorisés", géographiquement issus de territoires relevant de la politique de la ville,

- "Égalité des Chances - Femmes/Hommes", destinée à promouvoir les vocations scientifiques féminines.

Elles ont été initiées en 1999 et ont reçu le soutien constant des instances académiques, sésame indispensable pour travailler en partenariat avec des collèges et des lycées.

\subsection{Le programme Egalité des chances - Ouverture sociale}

Ce programme a un double objectif :

- Inciter à poursuivre des études supérieures afin d'accéder à des formations d'excellence",

- (Re)donner le goût des sciences et des technologies.

Il s'adresse à des élèves de collèges et de lycées de zones d'éduction prioritaire (ZEP), de zones urbaines sensibles (ZUS) ou de zones rurales de Lorraine.

Une importance est donnée à la formation de l'individu par la transmission de valeurs citoyennes et par le contact régulier avec des élèves-ingénieurs. Des visites de l'ESSTIN, d'autres établissements d'enseignement supérieur et d'entreprises, ainsi que des rencontres avec des ingénieurs et des acteurs économiques ont permis aux élèves de découvrir le monde professionnel.

Le projet comprend également un volet "culturel" incluant des visites de musées et des visites d'institutions.
Le contenu des thématiques abordées au cours de l'année est défini en lien avec les équipes pédagogiques des collèges et lycées partenaires.

Concrètement, les actions menées dans ce cadre consistent en :

- des séances de tutorat (2-3 h / semaine) consacrées à un travail méthodologique, à un "coaching" personnalisé, à des séances sur l'orientation scolaire, les sciences et le développement durable, ... De plus, les lycéens travaillent sur la communication et l'image de soi, alors que les collégiens participent à des TP scientifiques et technologiques (électrostatique, optique, motorisation, mécanique des fluides, ...) conçus par les étudiants de l'ESSTIN.

- la participation à des concours scientificotechnologiques qui servent de prétextes à la découvertes des disciplines scientifiques à travers une compétition entre classes de collèges. Citons le concours "Un Pont Pour l'Avenir" [7] [8], organisé en 2006-2007 et 2008-2009 par la CDEFI (Conférence des Directeurs des Écoles Françaises d'Ingénieurs), le BNEI (Bureau National des Élèves Ingénieurs) avec le soutien du CNISF (Conseil National des Ingénieurs et Scientifiques de France) autour de la construction de maquettes de ponts à base de spaghettis ou encore le concours "Course en Cours" [9] organisé par l'association éponyme avec le soutien de partenaires industriels tels que Dassault Systèmes et Renault qui a pour objectifs, la conception, la fabrication et la mise en compétition d'une mini voiture de course.

- des voyages de découverte (Paris, Strasbourg, ...)

- des visites d'entreprises (2 par an)

- des visites de Grandes Écoles et de laboratoires universitaires (2 par an)

- des visites culturelles (musées, théâtres, Parlement européen, Tribunal de Grande Instance, etc.)

\subsection{Le programme Egalité des chances - Femmes/Hommes}

Une équipe constituée d'enseignantes et d'étudiantes de l'école s'investit dans un programme en direction des jeunes femmes.

En effet, bien que plus présentes dans les filières scientifiques avant le baccalauréat, les filles, de part leur tendance à "s'autocensurer" et à se diriger vers certaines carrières à vocation plus altruiste, sont peu présentes dans les écoles d'ingénieurs. 
Ce programme vise à encourager des collégiennes et des lycéennes à poursuivre leurs études au sein de filières scientifiques et technologiques et à envisager des carrières dans ces domaines.

Pour favoriser les vocations scientifiques féminines et convaincre les jeunes femmes qu'elles aussi peuvent allier vie personnelle et vie professionnelle dans les domaines scientifiques et technologiques, diverses actions ont été mises en œuvre :

- interventions dans des collèges et des lycées de la région Lorraine et incitation à présenter des candidatures au Prix de la vocation scientifique et technique des filles [10]

- participation aux rencontres de l'association "Elles Bougent" [11] et au rallye Eco-mobilité Paris-Lyon-Marseille

- visites d'entreprises au sein desquelles les femmes ont un rôle prépondérant

- voyages de découverte (participation au Forum Air et Espace, visite du Musée des Arts et Métiers, du Musée de l'Air et de l'Espace, ...)

- organisation des Journées "Émilie du Châtelet" (31 janvier 2007, 26 février 2009 et 3 juin 2010) et du Prix "Caroline Aigle" (lancement en novembre 2009 dans l'académie de NancyMetz) du nom de la première femme brevetée pilote de chasse dans l'armée de l'air, décédée en 2007. Ce concours est ouvert aux lycéennes de seconde, première et terminale de l'académie de Nancy-Metz, qui doivent rédiger un texte autour d'un thème particulier. A titre d'exemple le sujet 2008 était "En 2019, vous êtes une jeune femme parfaitement accomplie, dont le parcours personnel et professionnel intéresse une journaliste. Écrivez l'article, d'un nombre maximum de 350 mots, qui peut prendre la forme d'une interview" ; le thème de l'édition 2011 est "Imaginez le premier discours de la première femme à avoir été nommée à un poste à très hautes responsabilités (en politique, en entreprise, dans l'administration, dans l'armée). Elle y évoque son parcours (familial et scolaire), les difficultés auxquelles elle a eu à faire face (réticences de son entourage, réactions de ses pairs masculins, conciliation de sa vie privée et de sa vie professionnelle ou publique), les encouragements dont elle a bénéficié, et les satisfactions que ses choix lui ont apportées, sans oublier ses perspectives pour l'avenir".

- conception et diffusion de la plaquette "Devenir ingénieurE : Se former en Lorraine" en collaboration avec les 16 écoles d'ingénieurs de Lorraine.

\section{Bilan quantitatif et qualitatif}

Le tableau ci-dessous quantifie les acteurs de la saison 2009-2010.

\begin{tabular}{|l|c|c|c|c|}
\hline & $\begin{array}{c}\text { Nombre } \\
\text { d'établissements } \\
\text { partenaires }\end{array}$ & $\begin{array}{c}\text { Nombre d'élèves } \\
\text { tutorés }\end{array}$ & $\begin{array}{c}\text { Nombre d'heures } \\
\text { de tutorat par élève }\end{array}$ & $\begin{array}{c}\text { Nombre d'élèves de } \\
\text { l'ESSTIN engagés }\end{array}$ \\
\hline Ouverture sociale & $\begin{array}{c}9 \text { collèges } \\
3 \text { lycées }\end{array}$ & $\begin{array}{c}437 \text { lycéens et } \\
\text { collégiens }\end{array}$ & $\sim 100 \mathrm{~h} /$ an & 60 \\
\hline Femmes/Hommes & $\begin{array}{c}4 \text { collèges } \\
31 \text { lycées }\end{array}$ & $\begin{array}{c}250 \text { lycéennes et } \\
\text { collégiennes }\end{array}$ & $\begin{array}{c}1 \text { journée } / \text { an } \\
\sim 20 \mathrm{~h} / \text { an }\end{array}$ & 11 \\
\hline
\end{tabular}

fig. 3 : bilan quantitatif

\section{Témoignages}

\subsection{Une enseignante du collège Claude Le Lorrain (Nancy)}

Dans le cadre d'un partenariat entre le collège Claude le Lorrain et l'ESSTIN autour d'un projet "Egalité des chances", des étudiants de l'école d'ingénieurs de Nancy ont mis en place et animé des ateliers hebdomadaires auprès de deux classes d'élèves de quatrième.

Au travers de travaux pratiques autour de thèmes divers - aérodynamisme, réalisation de fusées à eau, expériences sur les ondes et les fréquences -, ces étudiants ont fait découvrir aux élèves leur école (visite de l'ESSTIN) et le déroulement de leurs études en tentant de leur montrer l'importance $d u$ travail scolaire et de leur donner une ambition de réussite en dépit de leurs origines sociales modestes. Ils ont pu aussi mettre au service des élèves leurs compétences lors de la visite d'une scierie fonctionnant à la force de l'eau.

\subsection{L'équipe pédagogique du Collège Haut de Penoy (Vandœuvre lès Nancy)}


Ce sont des étudiants motivés, ponctuels qui ont pris leur rôle auprès des élèves très au sérieux. Ils ont pensé un projet global nouveau et personnalisé avec des Travaux Pratiques qui étaient cohérents. Les phases de manipulation et d'explication/correction ont été équilibrées. Au début, trop proches des élèves, les étudiants ont su s'adapter progressivement et mettre la distance nécessaire entre eux et les élèves pour le bon déroulement des séances.

Les documents (questionnaires, tutoriels ...) étaient bien adaptés et compréhensibles par les élèves. Les notions, bien que complexes, ont été abordées de façon ludique. Nous avons fortement apprécié le sérieux de l'organisation et la gestion des collégiens lors du voyage. Le document support $d u$ voyage était de qualité. Les étudiants de l'ESSTIN ont su intéresser les élèves aussi bien au niveau scientifique que culturel.

\subsection{Une étudiante actuellement à l'ESSTIN}

Trop de disparités subsistent dans les parcours scolaires des filles et des garçons. Un objectif ambitieux : plus de filles en filières scientifiques! C'est pourquoi le projet "Promouvoir les vocations scientifiques et techniques auprès des jeunes filles" a vu le jour. Notre but est de donner de l'ambition aux jeunes filles de collèges et lycées à l'aide de différents moyens de communication (conférences, débats, concours Caroline Aigle, salons,...). Le rôle des étudiantes est de prendre la place de grande sœur pour témoigner de leur parcours et montrer que "Quand on veut, on peut".

\subsection{Un ingénieur ESSTIN, tuteur au lycée Callot de 2006 à 2009 et vice-président de l'association nationale PARTAGE [13]}

Engagé dans le programme "PQPM Une grande école: PourQuoi Pas Moi ?" [12] de l'ESSTIN de 2006 à 2009 en tant que tuteur puis comme coordinateur-tuteur, j'ai trouvé dans ce partenariat un véritable développement personnel. Plus qu'un simple accompagnement, j'évoquerais davantage une aventure humaine aussi bien pour les tuteurs que pour leurs tutorés et une véritable formation citoyenne offerte en parallèle de notre cursus.

A travers un suivi au plus près des élèves, nous visons à les guider et à leur donner tous les éléments leur permettant d'atteindre au mieux leurs désirs de réussite dans la voie qu'ils auront choisie. Avec des séances de tutorat riches et aux thèmes variés, associées à des sorties culturelles, de véritables moments d'échanges et de partage ont lieu sur toute la durée de cet accompagnement. En trois années, l'évolution des jeunes est surprenante que ce soit aussi bien sur leur personnalité que sur leur soif de réussite. Leur motivation aussi est intense puisque ces jeunes font preuve de beaucoup de courage pour allier à la fois leurs études et un programme plutôt conséquent. Ils arrivent en tant qu' "enfants" dans ce programme et repartent en qualité de petit frère/petite sour à nos yeux et leurs aînés sont plus que fiers d'eux.

Mais du côté des tuteurs, qui seront les managers de demain, l'impact est aussi fort. En effet, ce programme est une formidable plate-forme pour une prise de conscience des problématiques actuelles de notre société que sont l'égalité des chances et la diversité. Il contribue également au développement et à l'enrichissement personnel de chacun de ses acteurs.

Nous savons qu'il reste encore beaucoup à faire pour passer à une réelle égalité des chances dans les faits, nous en sommes tous conscients, et essayons à travers ce programme d'apporter une pierre à l'édifice de la promotion de ces notions d'égalité des chances et de diversité. Ayant pour la plupart d'entre nous eu un parcours favorisé en partie par notre milieu social, nous nous devons de faire profiter de notre expérience tous ces jeunes pleins d'ambition pour qu'enfin le monde du partage remplace le partage du monde.

Preuve de la dynamique nationale qui se crée et où près de 1000 étudiants-tuteurs de 40 grandes écoles de commerce et d'ingénieurs suivent plus de 2500 lycéens actuellement, l'association nationale PARTAGE vient aujourd'hui en complémentarité et en continuité de PQPM d'une part en fédérant et en animant le réseau national de tuteur et, d'autre part, en proposant un suivi post-bac aux jeunes issus de ces programmes.

\section{Conclusions, Perspectives}

Le programme "Egalité des chances" de l'ESSTIN s'inscrit dans le prolongement de la Charte pour l'Égalité des Chances dans l'Accès aux Formations d'Excellence ; il a également été labellisé "Cordées de la réussite" et "PQPM" en 2009 ; certaines actions succinctement présentées supra sont détaillées via le site du Projet Egalité des Chances, géré par les étudiants www.egalite-deschances.com.

Dans le cadre national des Journées de l'Excellence et de la Réussite pilotées par les Ministères de l'Education Nationale et de l'Enseignement Supérieur, le Secrétariat d'Etat à la Politique de la Ville et le Commissariat à la Diversité et à l'Egalité des Chances [14], la journée du 30 janvier 2010 organisée à l'ESSTIN a permis de présenter les actions menées en Lorraine par les établissements d'enseignement supérieur et les entreprises pour donner à des collégiens et lycéens de l'ambition et l'envie d'accéder aux formations d'excellence. L'ESSTIN vient d'instaurer à la rentrée 2010 une 
"troisième voie" d'accès à l'école à travers des conventions de partenariat avec des lycées du Grand Est pour des élèves prometteurs issus de milieux défavorisés.

\section{Remerciements}

Les auteurs remercient l'ensemble des élèves, personnels et enseignants de l'ESSTIN, les collègues enseignants des collèges et lycées qui se sont impliqués dans ces actions, ainsi que les partenaires publics ou privés qui ont permis leurs concrétisations.

\section{Bibliographie et Webographie}

[1] Benoît Floc'h, Le Monde, 8 janvier 2010, "les grandes écoles face au procès de l'élitisme".

[2] Patrick Fauconnier et Jacqueline de Linares, le Nouvel Observateur, $n^{\circ} 2358 d u 14$ janvier 2010, Grandes Ecoles : comment rester entre soi.

[3] Repères et références statistiques - édition 2009, Ministère de l'Education Nationale, 426 pages, ISSN 1635 9089, ISBN 978-2-11097805-9.

[4] Filles et garçons sur le chemin de l'égalité, de l'école à l'enseignement supérieur, mars 2007, 35 pages, MESR, Direction générale de l'enseignement scolaire, Direction de l'évaluation, de la prospective, et de la performance.

[5] Pierre Bourdieu, Jean-Claude Passeron Les Héritiers, Les étudiants et la culture, 1964, Les éditions de minuit, Collection "Le sens commun", 192 pages, ISBN : 2.7073.0081.0, Nouvelle édition augmentée, 1966, 1985.

[6] Louis Gruel, Olivier Galland, Guillaume Houzel, Les étudiants en France, Histoire et sociologie d'une nouvelle jeunesse, Presses Universitaires de Rennes, collection le Sens Social, ISBN 978-2-7535-0970-2, novembre 2009.

[7] M. Robert, G. Lahon Grimaud, Établir de nouvelles passerelles entre enseignement supérieur et enseignement scolaire : le projet "Un pont pour l'avenir", Colloque National ASTEP 5-6 décembre 2007, Nantes, Comptes rendus des contributions, pp. 97-98-99.

[8] www.unpontpourlavenir.org

[9] Www.course-en-cours.com

[10] www.solidarite.gouv.fr/espaces, $770 /$ femmesegalite, 772/zoom-sur, $829 /$ nossites, 2001/espaces, $770 /$ femmesegalite, $772 /$ dossiers, 773/egaliteprofessionnelle, $1720 /$ promotion-de-1egalite, 896/le-prix-de-la-vocation, 7555.html

[11] www.ellesbougent.com

[12] www.pourquoipasmoi.essec.fr

[13] www.partage-interecoles.com

[14] eduscol.education.fr/cid50320/journees-de-1excellence-et-de-la-reussite.html

[15] Les écoles d'ingénieurs publiques et privées entre les rentrées 1988 et 2008, février 2011, 6 pages, MESR, DGESIP, DEPP, Note d'information 11-02, ISSN 2108-4033. 